\title{
Study on the relation between ground motion parameters and simulated earthquake damage of simplified masonry structures
}

\author{
TAO Dongwang ${ }^{1, a}$, CAI Yinliang ${ }^{1}$ \\ ${ }^{1}$ Key Laboratory of Earthquake Engineering and Engineering Vibration, Institute of Engineering \\ Mechanics, China Earthquake Administration, Harbin 150080, China \\ ataodongwang01@163.com
}

\begin{abstract}
Keywords: Ground motion parameter; Earthquake damage; Masonry structure; Damage index; Statistics analysis; Restoring force model
\end{abstract}

Abstract: Choosing proper earthquake ground motion parameters is important to establish a relation between strong ground motion and the earthquake damage. In this paper the statistical relationship between ten ground motion parameters and simulated mean earthquake damage indices of simplified masonry structures is investigated. The ten parameters to be used include time domain peaks and mean values, frequency domain peaks, time durations. Based on the field investigation on the structural earthquake damage conducted in Deayang City, Guanyuan City, Jiange County and Jiangyou City, simplied masonary structures group are generated with main controlling parameters as ratio of different number of stories, ratio of earthquake resist design. To simulate the earthquake damage of masonary structures under strong ground motion, simplied shear type model of masonary structures are used, with main controlling parameters as initial stiffness, first nonlinear stiffness, second nonlinear stiffness, yielding stress, ultimate stress. Using the 139 sets of strong ground motion in 12 earthquakes and thousands of simplied masonary structures, it is found that It is found that effective peak acceleration and peak ground acceleration which indicate the characteristics of force to structure have good relationship with damage of masonry structure, and the story number of masonry structure directly affects the strength of correlation between ground motion parameters and damage of masonry structure.

\section{Introduction}

In post earthquake damage assessment, the damage degree of single building is classified into five levels of damage ranks such as basic intactness, slight damage, moderate damage, serious damage and collapse with the help of the macro phenomenon of damage status of structures ${ }^{[1-2]}$. The average earthquake damage index indicates the weighted average of earthquake damage indices of various structures in a certain structural group and can be used to quantitatively evaluate the intensity of earthquake damage in a region. The earthquake damage is due to the strong ground motion under the basement of bulidings, and there are many parameters to exhibit the characteristics and damage potential of stong ground motions including time domain peaks and means, frequency domain peaks, and even time-frequency domain parameters ${ }^{[3]}$. It is important to analyze the relaiton between these parameters and earthquake damage. Since masonary structures classified as Type A, B and C are mainly used to damage investigation and damage assessment, this paper uses the investigation data of actual seismic damage in Sichuan area during the Wenchuan earthquake, and establishes a sets of nonlinear model group of masonary structures. The nonlinear seismic response of the structures under the actual ground motions are analyzed by elasto-plastic dynamic response analysis. Using the structural damage index and damage level to obtain the average earthquake damage index of the virtual structure groups at a given station area and to study the correlation between the ground motion parameters and the earthquake damage, strong ground motion parameters with good correlation with simulated earthquake damage of simplified masonary structures using statistical method are chosen for further research such as seismic intensity rapid report. 


\section{Strong Ground Motions}

Ground motion results of seismic waves released by the earthquake source, and the ground motion parameters mainly include time domain peaks, frequency spectrum and time duration. The time domain peaks include peak ground acceleration (PGA), peak ground velocity (PGV), peak ground displacement (PGD), the single directional PGA, the horizontal PGA, the combined PGA. The time domain means include root mean square of accelerations, Arias intensity ${ }^{[4]}$. The frequency domain peaks include peak frequency of Fouier spectrum, peak amplitude of Fouier spectrum, acceleration response spectrum, velocity response spectrum, displacement response spectrum, effective peak acceleration, effective peak velocity, and the frequency domain means include spectral intensity such as Clough spectral intensity, Housner spectral intensity ${ }^{[5]}$. Time duration parameters include the bracket duration and energy duration. In this paper 139 sets of strong ground motion in 12 earthquakes are use list in Table 1.

Table 1. 12 The list of earthquake events and number of ground motions

\begin{tabular}{cccccc}
\hline Serial & Earthquake & $\begin{array}{c}\text { Epicenter } \\
\text { province }\end{array}$ & Time & Magnitude & Numbers \\
\hline 1 & Wenchuan & Sichuan & $2008-05-12$ & M8.0 & 87 \\
2 & Lushan & Sichuan & $2013-04-20$ & M7.0 & 12 \\
3 & Panzhihua & Sichuan & $2008-08-30$ & M6.1 & 4 \\
4 & Ninger & Yunan & $2007-06-03$ & M6.4 & 3 \\
5 & Yingjiang & Yunan & $2008-08-21$ & M5.8 & 1 \\
6 & Yaoan & Yunan & $2009-07-09$ & M6.0 & 1 \\
7 & Wuqia & Xinjiang & $2008-10-05$ & M6.8 & 3 \\
8 & Minxian & Gansu & $2013-07-22$ & M6.6 & 4 \\
9 & Ludian & Yunan & $2014-08-03$ & M6.5 & 8 \\
10 & Jinggu & Yunan & $2014-10-07$ & M6.6 & 5 \\
11 & Kangding & Sichuan & $2014-11-22$ & M6.3 & 3 \\
12 & Kangding & Sichuan & $2014-11-25$ & M5.8 & 8 \\
\hline
\end{tabular}

\section{Masonary Structures Catergory based on Field Investigaiton}

After Wenchuan earthquake, China Earthquake Administration organized several important field investigations on the structural earthquake damage. One team led by Professor Sun Jingjiang has macro seismic intensity survey and the masonary structures in the four cite are shown in Table $2.70 \%$ of the masonary structures are used for residence, followed by Government, Education and Business, and the number of stories is mainly in 3-6. The residence buildings are designed to withstand intensity VII ground motion after 1992.

Table 2. Number and ratio of different use of masonary structures in four cites

\begin{tabular}{ccccccc}
\hline Usage & Resident & Education & Mediation & Govern. & Business & Total \\
\hline Deyang & 13 & 1 & -- & 3 & 1 & 18 \\
Guanyuan & 83 & 3 & 4 & 5 & 12 & 107 \\
Jiange & 10 & 11 & 2 & 9 & -- & 32 \\
Jiangyou & 27 & -- & 2 & 3 & 1 & 33 \\
Sum & 133 & 15 & 8 & 20 & 14 & 190 \\
Ratio $(\%)$ & $70 \%$ & $8 \%$ & $4 \%$ & $11 \%$ & $7 \%$ & $100 \%$ \\
\hline
\end{tabular}

\section{Nonlinear Restoring Force Model for Masonary Structures}

Multistories masonary structures can be modelled as layer model, which layers the mass of each layer into the mass center of the floor cover and replaces the stiffness of all members in this layer with a total 
equivalent lateral stiffness, forming a vertical mass-series spring model. For simplicity and computer efficience, shear type layer model are implemented to model Multistories masonary structures. The inter story elements especially the weight resist wall of masonary structures undergo nonlinear behaviour under large ground motions, and trilinear force-displacement relation is used to model the nonlinear restoring force. The controlling parameters as initial stiffness $K_{0}$, first nonlinear stiffness $K_{1}$, second nonlinear stiffness $K_{2}$, yielding stress $Q \mathrm{y}$, ultimate stress $Q \mathrm{u}$ as shown in Figure $1^{[6]}$.

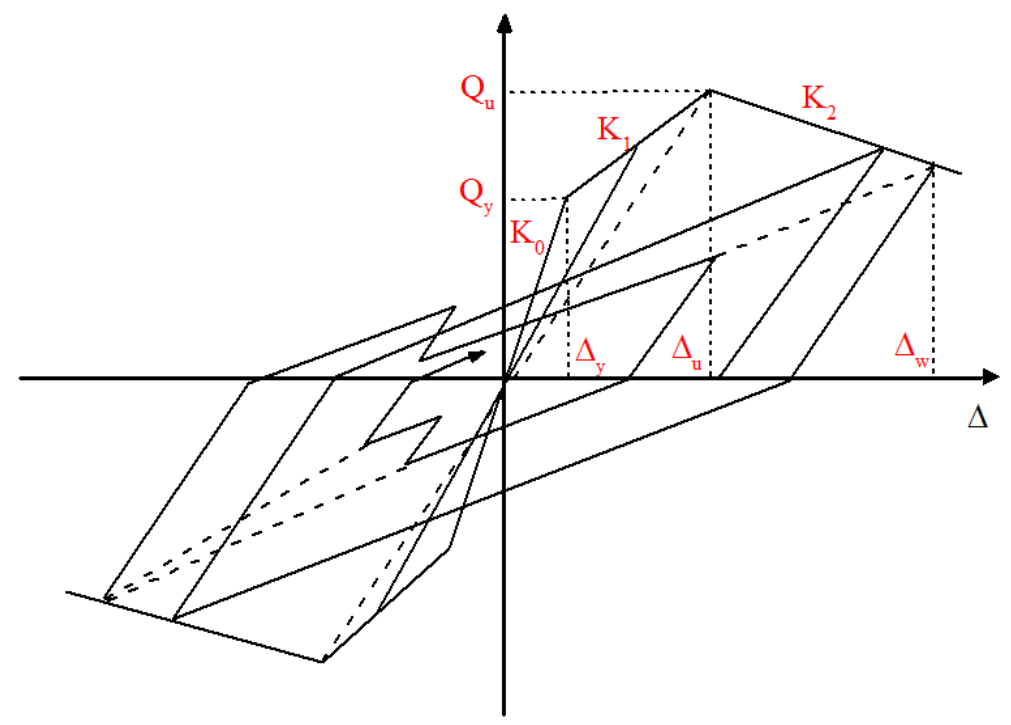

Figure 1. Restoring force model for masonary walls

The parameters $K_{2}, Q \mathrm{u}$ are dependant on the other three and the corresponding variablity are small, to simulated a group nonlinear model, the independent stochastic variabes are $K_{0}, K_{1}$ and $Q \mathrm{y}$, and the vairation coefficients are set as $0.3,0.33$ and 0.3 for each in respectively. For a masonary structure with givien stories, stratified sampling, each independent stochastic variabes taking 5 values within plus or minus one standard deviation of the average value of the sample respectively, is used to generate the interstory nonlinear model for each story, and large number of nonlinear a masonary structure are generated. A random combination method is adopted expanded 125 masonry structure nonlinear models. The other multistories masonary structures are also established.

\section{Earthquake Damage Index}

The damage degree of masonry structure is measured by the parameters of ductility coefficient given as Equation 1:

$$
\mu=\frac{x_{\max }}{x_{y}}
$$

where $x_{\max }$ is the maximal interstory displacement, and $x_{y}$ is the displacment when the walls begin to have crack. The masonry structure is classified as basic intactness when $\mu \leq 1$; basic intactness, slight damage when $1 \leq \mu \leq 2$; moderate damage when $2 \leq \mu \leq 4$; serious damage when $4 \leq \mu \leq 6$ and collapse when $\mu>6$.

The average earthquake damage index $D$ refers to the weighted average of the earthquake damage indices of similar buildings at a certain survey point, that is, the sum of the product of earthquake damage buildings at various damage levels or classes and its corresponding earthquake damage index, given by Equation $2^{[7]}$

$$
D=\sum_{i=1}^{5} d_{i} \lambda_{i}
$$

where $d_{i}$ is the damage index for damage level $i, \lambda_{i}$ is the damage ratio of building using the numbers or areas of damaged to the total buildings. The damage index is set as 0.05 for basic intactness; 0.2 for 
slight damage; 0.43 for moderate damage; 0.7 for serious damage and 0.93 for collapse. Different weights are assigned to masonry structure with different stories given by Table 3 .

Table 3. The weight coefficience for structure with different stoties

\begin{tabular}{cccccccc}
\hline Stories & 2 & 3 & 4 & 5 & 6 & 7 & Sum \\
\hline B Type & 0.06 & 0.21 & 0.21 & 0.29 & 0.21 & 0.02 & 1.00 \\
C Type & 0.04 & 0.10 & 0.05 & 0.02 & 0.65 & 0.14 & 1.00 \\
\hline
\end{tabular}

\section{Results}

Take the ground motion at Jiangyou Zhonghua station 051JYC in Wenchuan Earthquake for example, the calcuated 10 ground motion parameters are given in Table 4.

Table 4. Calcuated 10 ground motion parameters at station 051JYC

\begin{tabular}{ccccccccccc}
\hline Parameters & PGAR & PGVR & PSV & PSAs & PSVs & SIc & SI $_{\mathrm{h}}$ & PGAc & $\mathrm{A}_{03}$ & $\mathrm{~A}_{05}$ \\
\hline Values & 310.75 & 31.62 & 73.97 & 470.64 & 28.69 & 13.67 & 9.17 & 270.63 & 169.05 & 207.73 \\
\hline
\end{tabular}

Using Wilson- $\theta$ method to calculate the multi degree of freedom shear type nonlinear seismic response, the interstory force displaement for a 3-story building is shown in Figure 2.

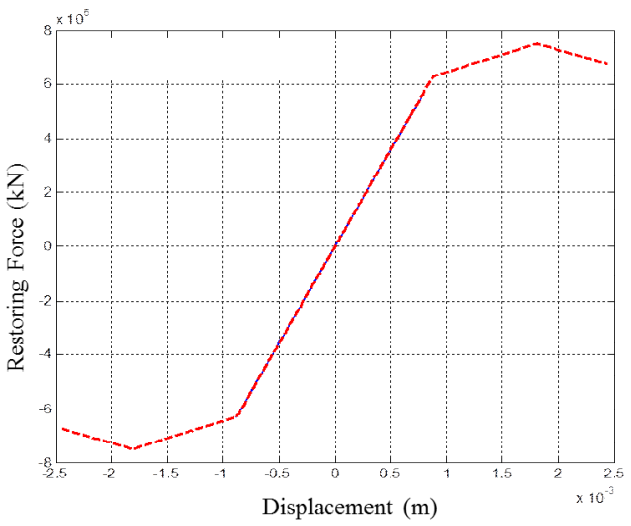

(a) Story 1

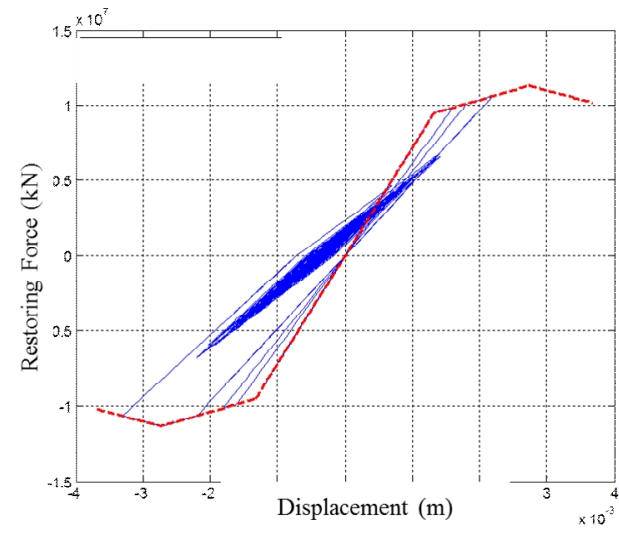

(b) Story 2

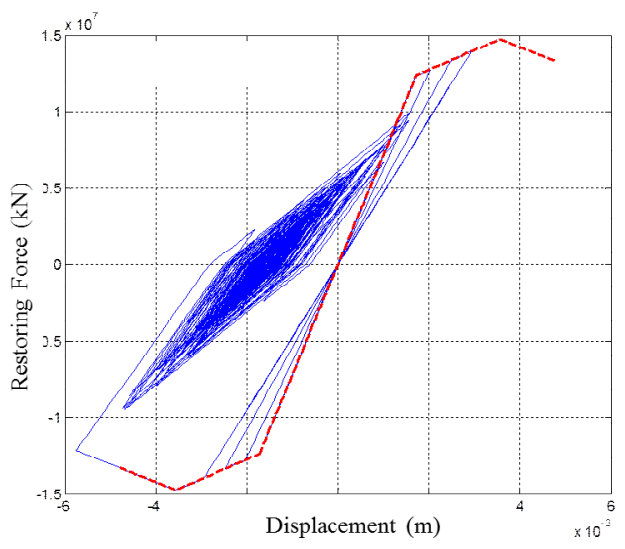

(c) Story 3

Figure 2. Nonlinear restoring force and displacement curves for a Type B strcuture (red line is the constitutive relation and the blue line is response relation)

Spearman rank correlation coefficient (SRCC)is used to study relation of the ground motion parameters and average earthquake damage index, as given by Equation $3^{[8]}$

$$
\rho_{s}=\frac{\sum\left(x_{i}^{\prime}-\bar{x}^{\prime}\right)\left(y_{i}^{\prime}-\bar{y}^{\prime}\right)}{\sqrt{\sum\left(x_{i}^{\prime}-\bar{x}^{\prime}\right)^{2} \sum\left(y_{i}^{\prime}-\bar{y}^{\prime}\right)^{2}}}
$$


The SRCCs for ground motion parameters and damge index are shown in Figure 3, for brevity only the results for PGA, PGV, PSA and $\mathrm{Si}_{\mathrm{h}}$ are shown here.

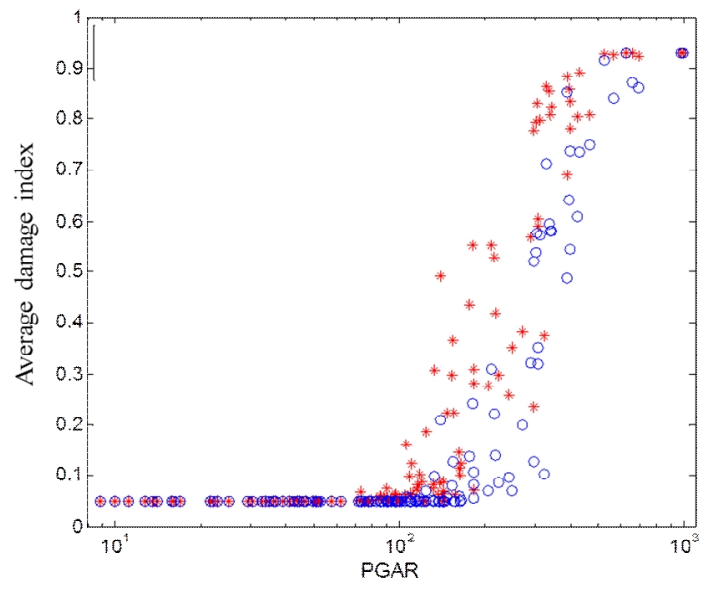

(a) PGA

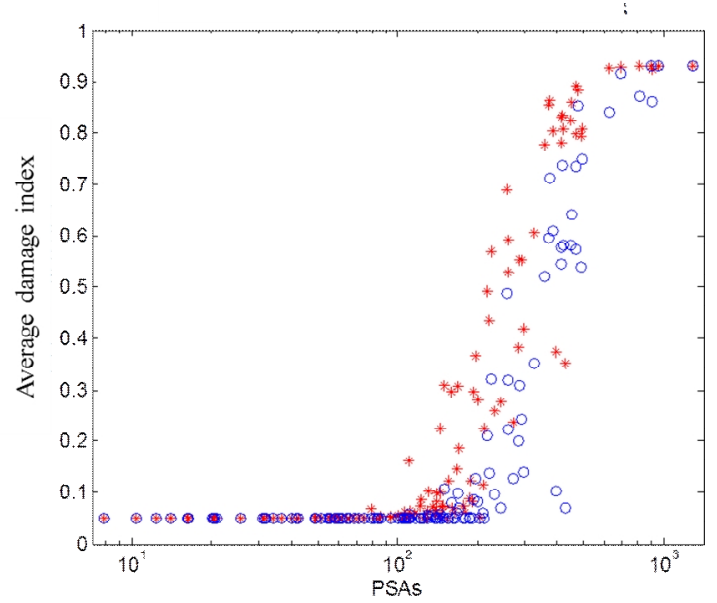

(c) PSA

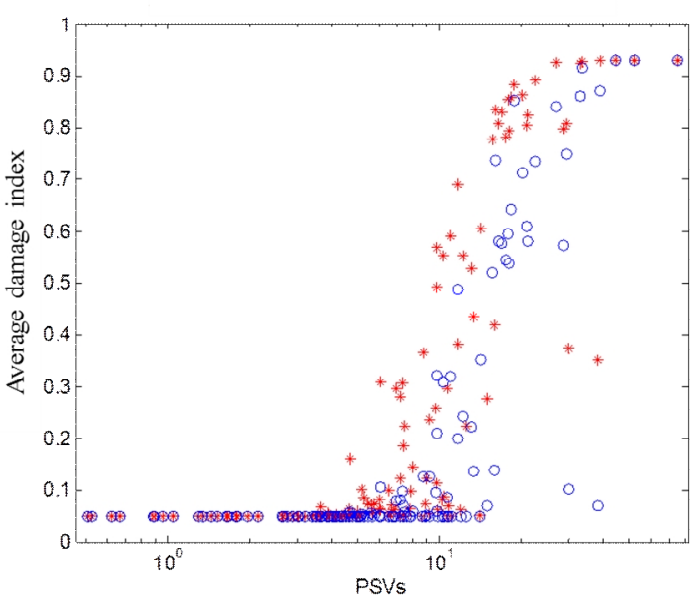

(b) PGV

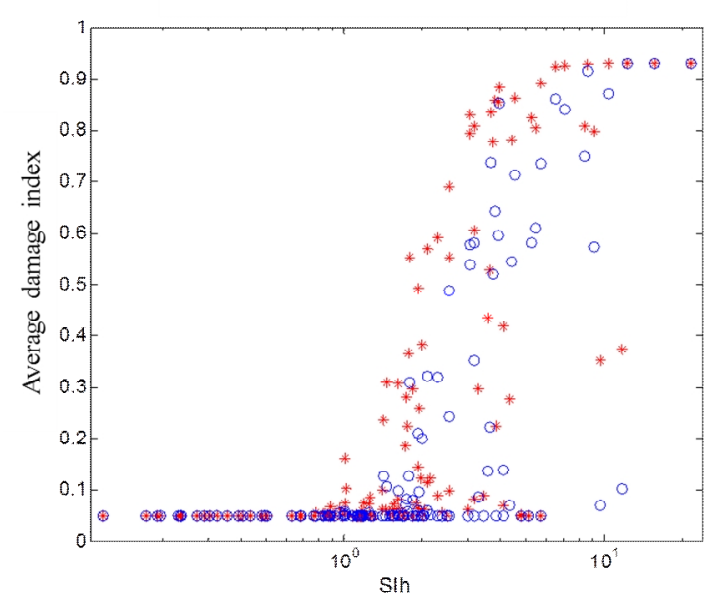

(d) $\mathrm{SI}_{\mathrm{h}}$

Figure 3. SRCCs of ground motion parameters and average damage index of masonary structures Nonlinear restoring force and displacement curves for a Type B strcuture

(red line is for Type B and the blue is for the Type C masonary structures)

It can be seen from Figure 3 and SRCCs results that the ten ground motion parameters have some correlations with the average damage index of masonry structure, and the SRCCs values for Type B masonry structure are all larger than 0.7 , and larger than 0.66 for Type $C$ buildings. The correlation between ground motion parameters and seismic damage of Type B structures is better than that of Type $\mathrm{C}$ structures. Within a certain range, with the increase level of ground motion parameters, the masonry structure is not damaged (the average damage index is a straight line); then the damage of the masonry structure increases with the increase level of ground motion parameters is a positive correlation; when the local seismic parameters reach a certain value, with the increase of ground motion parameters, the masonry structure begins to be completely damaged or collapsed (the average earthquake damage index is also close to a straight line). The correlations of the ten kinds of ground motion parameters that characterize the basic characteristics of ground motion and the earthquake intensity have little difference (the correlation range is between 0.67 and 0.70 ), and the correlation with the earthquake damage of masonry structures is somewhat different. The range of SRCC is between $0.66 \sim 0.91$ ). Among them, some of the most pertinent seismicity parameters are PGAs and PSAs, and the correlation between these two ground motion parameters is more than 0.96 . 


\section{Conclusion}

In this paper strong ground motions at 139 stations in 12 devastating earthquakes in China are used to excite simplified masonary structures whose nonlinear behavior is controlled by initial stiffness, first nonlinear stiffness and yielding stress, and the Spearman rank correlation coefficient between the 10 ground motion parameters and average earthquake damage index are calculated and compared. Through comparative analysis of the SRCCs, the parameters that have good correlation with the masonry structure are parameters that characterize the effect of ground motion on the structural force, such as PGAs and PSAs, which maybe should mainly in earthquake damage assessment and seismic intensity rapid reporting.

\section{Acknowledgements}

This work was supported by Scientific Research Fund of Institute of Engineering Mechanics, China Earthquake Administration (2016QJGJ21, 2016A03, 2014B08), National Natural Science Foundation of China (5150082083).

\section{References}

[1]. Li Shan-you, Jin Xing, Chen Xian, Ma Qiang. Rapid reporting of peak strong motion and seismic intensity [J]. Earthquake Engineering and Engineering Vibration, 22(6):1-7, 2002 (in Chinese).

[2]. U.S. Geological Survey (USGS). Earthquakes with 1,000 or more deaths since 1900, available at http://earthquake.usgs.gov/earthquakes/world/world_deaths.php, $2011 \mathrm{a}$.

[3]. Kaka S L, Atkinson G M. Relationships between instrumental ground-motion parameters and modified Mercalli intensity in eastern North America [J]. Bulletin of the Seismological Society of America, 2004, 94(5): 1728-1736.

[4]. Arias, A. A measure of earthquake intensity [J]. In Seismic Design for Nuclear Power Plants, Editor R. J. Hansen, MIT Press, Cambridge, MA, 1970, 438-483.

[5].Housner, G. Spectrum intensities of strong-motion earthquakes, Proceeding of the Symposium on Earthquake and Blast Effects on Structures [J]. Earthquake Engineering Research Institute, 1952, 20-36.

[6]. Zhang Lingxin, Jiang Jinren, Liu Jieping. Seismic vulnerability analysis of multistory dwelling brick buildings. Earthquake Engineering and Engineering Vibration [J], 22(1):49-55, 2002 (in Chinese).

[7]. Bo Jingshan, Zhang jianyi, Sun pingshan, Li Wei, Li Ping. Discussion on seismic damage index and relevant problems, Journal of Natural Disasters [J], 2012, 21(6):37-42 (in Chinese).

[8].Bonett DG, Wright, TA. Sample size requirements for Pearson, Kendall, and Spearman correlations. Psychometrika [J]. 2000, 65: 23-28. 